\title{
The existence of multiple positive solutions for a class of semipositone Dirichlet boundary value problems
}

\author{
Minling Zhong • Xinguang Zhang
}

Received: 20 April 2010 / Published online: 29 January 2011

(C) The Author(s) 2011. This article is published with open access at Springerlink.com

Abstract In this paper, we consider the existence of multiple positive solutions for the following singular semipositone Dirichlet boundary value problem:

$$
\left\{\begin{array}{l}
-x^{\prime \prime}(t)=p(t) f(t, x)+q(t), \quad t \in(0,1), \\
x(0)=0, \quad x(1)=0,
\end{array}\right.
$$

where $p:(0,1) \rightarrow[0,+\infty)$ and $f:[0,1] \times[0,+\infty) \rightarrow[0,+\infty)$ are continuous, $q:(0,1) \rightarrow(-\infty,+\infty)$ is Lebesgue integrable. Under certain local conditions and superlinear or sublinear conditions on $f$, by using the fixed point theorem, some sufficient conditions for the existence of multiple positive solutions are established for the case in which the nonlinearity is allowed to be sign-changing.

Keywords Singular boundary value problem - Semipositone $\cdot$ Positive solutions . Fixed point $\cdot$ Cone

Mathematics Subject Classification (2000) 34B15 · 34B25

\section{Introduction}

Nonlinear boundary value problems, as an important branch of modern applied mathematics, have been studied by many authors in recent years [1, 4, 7-11]. In the study

The second author is financially supported by the National Natural Science Foundation of China $(11071141,10771117)$ and the Natural Science Foundation of Shandong Province of China (Y2007A23, ZR2010AM017).

\section{Zhong}

School of Informatics, Guangdong University of Foreign Studies, Guangzhou 510006, China

X. Zhang $(\bowtie)$

School of Mathematical and Informational Sciences, Yantai University, Yantai 264005, China

e-mail: zxg123242@sohu.com 
of nonlinear boundary value problems, one often has to use the cone theory or upper and lower solution methods. To use these theories and methods, the nonlinearity of the boundary value problem has to be nonnegative and continuous. However, not all the nonlinearities of the boundary value problems arising from real world applications are nonnegative. If the nonlinearity may take on negative values, we call this type of problems as semipositone problems, which arise naturally in chemical reactor theory, design of suspension bridges, combustion and management of natural resources $[2,3,5]$.

Recently, Zhang [12] considered the following singular semipositone Dirichlet boundary value problem:

$$
\left\{\begin{array}{l}
x^{\prime \prime}(t)+f(t, x)+q(t)=0, \quad t \in(0,1), \\
x(0)=0, \quad x(1)=0,
\end{array}\right.
$$

where $f: C(0,1) \times[0,+\infty) \rightarrow[0,+\infty)$ is continuous, $q(t):(0,1) \rightarrow(-\infty,+\infty)$ is Lebesgue integrable. Under the superlinear condition, by using the fixed point index, the authors established the existence of positive solutions for the semipositone problem (1.1). But Zhang [12] did not establish the conditions for the existence of multiple positive solutions, and also did not consider the sublinear case. This paper fills this gap in the literature, i.e, we concern with the multiple solutions of the following singular semipositone Dirichlet boundary value problem:

$$
\left\{\begin{array}{l}
-x^{\prime \prime}(t)=p(t) f(t, x)+q(t), \quad 0<t<1, \\
x(0)=0, \quad x(1)=0,
\end{array}\right.
$$

where $p:(0,1) \rightarrow[0,+\infty)$ and $f:[0,1] \times[0,+\infty) \rightarrow[0,+\infty)$ are continuous, $q:(0,1) \rightarrow(-\infty,+\infty)$ is Lebesgue integrable. Under certain local conditions and superlinear or sublinear conditions on $f$, we find that there should at least exist two positive solutions for the semipositone problem (1.2). In addition, our method can also be applied to solve Sturm-Liouville boundary value problems or multi-point boundary value problems.

This paper is organized as follows. Section 2 gives some preliminaries and lemmas. Section 3 is devoted to the main results and their proof. At the end, two examples are given to demonstrate the application of our main results.

\section{Preliminaries and lemmas}

Definition 2.1 Let $E$ be a real Banach space. A nonempty closed convex set $P \subset E$ is called a cone of $E$ if it satisfies the following two conditions:

(1) For $x \in P, \lambda^{*} \geq 0$ implies $\lambda^{*} x \in P$;

(2) For $x \in P,-x \in P$ implies $x=\theta$.

Definition 2.2 An operator is called completely continuous if it is continuous and maps bounded sets into precompact sets. 
Lemma 2.1 [6] Let $E$ be a real Banach space, $P \subset E$ be a cone. Assume $\Omega_{1}, \Omega_{2}$ are two bounded open subsets of $E$ with $\theta \in \Omega_{1}, \bar{\Omega}_{1} \subset \Omega_{2}$, and let $T: P \cap\left(\bar{\Omega}_{2} \backslash \Omega_{1}\right) \rightarrow P$ be a completely continuous operator such that either

(1) $\|T x\| \leq\|x\|, x \in P \cap \partial \Omega_{1}$ and $\|T x\| \geq\|x\|, x \in P \cap \partial \Omega_{2}$, or

(2) $\|T x\| \geq\|x\|, x \in P \cap \partial \Omega_{1}$ and $\|T x\| \leq\|x\|, x \in P \cap \partial \Omega_{2}$.

Then $T$ has a fixed point in $P \cap\left(\bar{\Omega}_{2} \backslash \Omega_{1}\right)$.

Let $G(t, s)$ be the Green's function for the following boundary value problem

$$
\left\{\begin{array}{l}
-x^{\prime \prime}=0, \quad 0<t<1, \\
x(0)=0, \quad x(1)=0,
\end{array}\right.
$$

then

$$
G(t, s)= \begin{cases}s(1-t), & 0 \leq s \leq t \leq 1 \\ t(1-s), & 0 \leq t \leq s \leq 1\end{cases}
$$

Remark 2.1 Clearly, for any $t, s \in[0,1]$, we have

$$
t(1-t) s(1-s) \leq G(t, s) \leq s(1-s) \quad \text { or } \quad t(1-t) .
$$

In the rest of the paper, we adopt the following assumptions:

(H1) $f:[0,1] \times[0,+\infty) \rightarrow[0,+\infty)$ is continuous.

(H2) $p:(0,1) \rightarrow[0,+\infty)$ is continuous and $q:(0,1) \rightarrow(-\infty,+\infty)$ is Lebesgue integrable, such that $\int_{0}^{1} q_{-}(s) d s=r>0$ and

$$
0<\int_{0}^{1} s(1-s)\left[p(s)+q_{+}(s)\right] d s=L<2 r,
$$

where $q_{+}(t)=\max \{q(t), 0\}, q_{-}(t)=\max \{-q(t), 0\}$.

(H3) For any $(t, x) \in[0,1] \times[0,2 r]$,

$$
f(t, x) \leq \frac{2 r}{L}-1
$$

(H4) There exists a constant $R>2 r$ such that, for any $(t, x) \in[0,1] \times\left[\frac{3}{32} R, R\right]$,

$$
f(t, x) \geq \frac{16 R}{3 l}
$$

where $l=\int_{\frac{1}{4}}^{\frac{3}{4}} s(1-s) p(s) d s$.

(H5)

$$
\lim _{x \rightarrow+\infty} \frac{f(t, x)}{x}=0
$$

for $t$ uniformly on [0, 1]. 
(H6)

$$
\lim _{x \rightarrow+\infty} \frac{f(t, x)}{x}=+\infty
$$

for $t$ uniformly holds on any close subinterval of $(0,1)$.

Now let us consider the Banach space $E=C[0,1]$ equipped with the standard norm

$$
\|x\|=\max _{0 \leq t \leq 1} x(t)
$$

Set

$$
C^{+}[0,1]=\{x \mid x(t) \geq 0, t \in[0,1]\}, \quad P=\left\{x \in C^{+}[0,1]: x(t) \geq t(1-t)|x|\right\},
$$

then $P$ is a cone of $E$.

Consider the linear boundary value problem

$$
\left\{\begin{array}{l}
w^{\prime \prime}(t)+q_{-}(t)=0, \quad t \in(0,1) \\
w(0)=0, \quad w(1)=0
\end{array}\right.
$$

By (H2) and (2.1), the problem (2.3) has unique positive solution

$$
w(t)=\int_{0}^{1} G(t, s) q_{-}(s) d s, \quad 0 \leq t \leq 1 .
$$

Define the function, for $u \in C[0,1]$,

$$
[u(t)]^{*}= \begin{cases}u(t), & u(t) \geq 0, \\ 0, & u(t)<0 .\end{cases}
$$

Let us consider the following approximately singular nonlinear boundary value problem

$$
\left\{\begin{array}{l}
z^{\prime \prime}(t)+p(t) f\left(t,[z(t)-w(t)]^{*}\right)+q_{+}(t)=0, \quad t \in(0,1), \\
z(0)=0, \quad z(1)=0 .
\end{array}\right.
$$

Lemma 2.2 If $\tilde{z}_{0}$, satisfying $\tilde{z}_{0}(t) \geq w(t)$ for any $t \in[0,1]$, is a unique positive solution of the $B V P(2.4)$, then $x_{0}(t)=\tilde{z}_{0}(t)-w(t)$ is a positive solution of the $B V P$ (1.2).

Proof In fact, if $\tilde{z}_{0}$ is a positive solution of (2.4) such that $\tilde{z}_{0}(t) \geq w(t)$ for any $t \in$ $[0,1]$. Let $x_{0}(t)=\tilde{z}_{0}(t)-w(t)$, then $x_{0}(t) \geq 0, t \in[0,1]$ (positive on $(0,1)$ ). Since $w(t)$ is unique positive solution of (2.3), for any $t \in[0,1]$, we have

$$
\tilde{z}_{0}^{\prime \prime}(t)+p(t) f\left(t, \tilde{z}_{0}(t)-w(t)\right)+q_{+}(t)=0,
$$


i.e.,

$$
x_{0}^{\prime \prime}(t)+w^{\prime \prime}(t)+p(t) f\left(t, x_{0}(t)\right)+q_{+}(t)=0 .
$$

It follows from

$$
x_{0}^{\prime \prime}(t)+p(t) f\left(t, x_{0}(t)\right)+q_{+}(t)-q_{-}(t)=0,
$$

that

$$
x_{0}^{\prime \prime}(t)+p(t) f\left(t, x_{0}(t)\right)+q(t)=0 .
$$

On the other hand, clearly

$$
x_{0}(0)=\tilde{z}_{0}(0)-w(0)=0, \quad x_{0}(1)=\tilde{z}_{0}(1)-w(1)=0 .
$$

These guarantee that $x_{0}$ is positive solution of the BVP (1.2).

Now define an integral operator $T: P \rightarrow C[0,1]$ by

$$
(T z)(t)=\int_{0}^{1} G(t, s)\left[p(t) f\left(s,[z(s)-w(s)]^{*}\right)+q_{+}(s)\right] d s .
$$

Lemma 2.3 Assume that (H1) and (H2) hold. Then $T(P) \subset P$ and $T: P \rightarrow P$ is a completely continuous operator.

Proof For any $z \in P, t \in[0,1]$, by (2.1), we have

$$
(T z)(t) \leq \int_{0}^{1} s(1-s)\left[p(t) f\left(s,[z(s)-w(s)]^{*}\right)+q_{+}(s)\right] d s,
$$

and thus

$$
\|T z\| \leq \int_{0}^{1} s(1-s)\left[p(t) f\left(s,[z(s)-w(s)]^{*}\right)+q_{+}(s)\right] d s .
$$

On the other hand, it follows from (2.1) that

$$
(T z)(t) \geq t(1-t) \int_{0}^{1} s(1-s)\left[p(t) f\left(s,[z(s)-w(s)]^{*}\right)+q_{+}(s)\right] d s,
$$

which implies

$$
(T z)(t) \geq t(1-t)\|T z\|
$$

Therefore $T(P) \subset P$.

Let $B \subset P$ be any bounded set, then, for any $z \in B$, there exists a constant $M>0$ such that $\|z\| \leq M$. Thus, for any $z \in B, s \in[0,1]$, noticing that $[z(s)-w(s)]^{*} \leq$ $z(s) \leq\|z\| \leq M$, we have

$$
|(T z)(t)|=\int_{0}^{1} G(t, s)\left[p(t) f\left(s,[z(s)-w(s)]^{*}\right)+q_{+}(s)\right] d s
$$




$$
\begin{aligned}
& \leq \int_{0}^{1} s(1-s)\left[\max _{[0,1] \times[0, M]} f(t, x)+1\right]\left[p(s)+q_{+}(s)\right] d s \\
& \leq\left[\max _{[0,1] \times[0, M]} f(t, x)+1\right] \int_{0}^{1} s(1-s)\left[p(s)+q_{+}(s)\right] d s \\
& <+\infty .
\end{aligned}
$$

Therefore $T(B)$ is uniformly bounded.

Now we show that $T(B)$ is equicontinuous on $[0,1]$. For any $z \in B, t \in[0,1]$, we have

$$
\begin{aligned}
\left|\frac{d}{d t}(T z)(t)\right|= & \mid-\int_{0}^{t} s\left[p(s) f\left(s,[z(s)-w(s)]^{*}\right)+q_{+}(s)\right] d s \\
& +\int_{t}^{1}(1-s)\left[p(s) f\left(s,[z(s)-w(s)]^{*}\right)+q_{+}(s)\right] d s \mid \\
\leq & {\left[\max _{[0,1] \times[0, M]} f(t, x)+1\right]\left(\int_{0}^{t} s\left[p(s)+q_{+}(s)\right] d s\right.} \\
& \left.+\int_{1}^{t}(1-s)\left[p(s)+q_{+}(s)\right] d s\right) .
\end{aligned}
$$

By changing the order of integration, we obtain

$$
\begin{aligned}
& \int_{0}^{1}\left(\int_{0}^{t} s\left[p(s)+q_{+}(s)\right] d s+\int_{t}^{1}(1-s)\left[p(s)+q_{+}(s)\right] d s\right) d t \\
& \quad=\int_{0}^{1} d s \int_{s}^{1} s\left[p(s)+q_{+}(s)\right] d t+\int_{0}^{1} d s \int_{0}^{s}(1-s)\left[p(s)+q_{+}(s)\right] d t \\
& \quad=2 \int_{0}^{1} s(1-s)\left[p(s)+q_{+}(s)\right] d s<+\infty .
\end{aligned}
$$

So for any $z \in B$, we have

$$
\begin{aligned}
0 & \leq \int_{0}^{1}\left|\frac{d}{d t}(T z)(t)\right| d t \leq 2\left[\max _{[0,1] \times[0, M]} f(t, x)+1\right] \int_{0}^{1} s(1-s)\left[p(s)+q_{+}(s)\right] d s \\
& <+\infty
\end{aligned}
$$

From the absolute continuity of the integral, we know that $T(P)$ is equicontinuous on $[0,1]$. Thus according to the Ascoli-Arzela Theorem, $T(P)$ is a relatively compact set. Now, by the continuity of $f$ and the Lebesgue control convergence theorem, it is easy to know that $T: P \rightarrow P$ is continuous. Thus $T$ is a completely continuous operator. 


\section{Main results}

Theorem 3.1 Suppose (H1)-(H5) hold. Then the BVP (1.2) has at least two positive solutions $x_{1}(t)$ and $x_{2}(t)$ such that $x_{1}(t) \geq r t(1-t), x_{0}(t) \geq \frac{1}{2} \operatorname{Rt}(1-t), t \in[0,1]$, where $r$ and $R$ are defined by (2.2) and (H4), respectively.

Proof Let $\Omega_{r}=\{z \in P:\|z\|<2 r\}$ and $\partial \Omega_{r}=\{z \in P:\|z\|=2 r\}$. Then, for any $z \in \partial \Omega_{r}, s \in[0,1]$, we have

$$
0 \leq[z(s)-w(s)]^{*} \leq z(s) \leq\|z\| \leq 2 r .
$$

It follows from $(\mathbf{H 3})$ that

$$
\begin{aligned}
\|T z\| & \left.=\max _{t \in[0,1]} \int_{0}^{1} G(t, s)\left[p(s) f\left(s,[z(s)-w(s)]^{*}\right)\right)+q_{+}(s)\right] d s \\
& \leq \int_{0}^{1} s(1-s)\left[\left(\frac{2 r}{L}-1\right) p(s)+q_{+}(s)\right] d s \\
& \leq \frac{2 r}{L} \int_{0}^{1} s(1-s)\left[p(s)+q_{+}(s)\right] d s \\
& =2 r=\|z\| .
\end{aligned}
$$

Therefore, $\|T z\| \leq\|z\|, z \in P \cap \partial \Omega_{r}$.

On the other hand, let $\Omega_{R}=\{z \in P:\|z\|<R\}$ and $\partial \Omega_{R}=\{z \in P:\|z\|=R\}$. Then for any $z \in \partial \Omega_{R}, t \in\left[\frac{1}{4}, \frac{3}{4}\right]$, noticing $R>2 r$, we have

$$
\begin{aligned}
z(t)-w(t) & \geq z(t)-t(1-t) \int_{0}^{1} q_{-}(s) d s=z(t)-t(1-t) r \\
& \geq z(t)-z(t)\|z\| r=z(t)-\frac{r}{R} z(t) \geq \frac{1}{2} z(t) \\
& \geq \frac{1}{2} t(1-t)\|z\| \geq \frac{3}{32} R>0 .
\end{aligned}
$$

So for any $z \in \partial \Omega_{R}, t \in\left[\frac{1}{4}, \frac{3}{4}\right]$, we have

$$
\frac{3}{32} R \leq z(t)-w(t) \leq R
$$


It follows from (H4) and (3.2) that, for any $z \in \partial \Omega_{R}, t \in\left[\frac{1}{4}, \frac{3}{4}\right]$,

$$
\begin{aligned}
\|T z\| \geq(T z)(t) & =\int_{0}^{1} G(t, s)\left[p(s) f\left(s,[z(s)-x(s)]^{*}+q_{+}(s)\right] d s\right. \\
& \geq \int_{\frac{1}{4}}^{\frac{3}{4}} G(t, s)\left[p(s) f(s, z(s)-x(s))+q_{+}(s)\right] d s \\
& \geq \frac{16 R}{3 l} \int_{\frac{1}{4}}^{\frac{3}{4}} G(t, s) p(s) d s \geq \frac{16 R}{3 l} t(1-t) \int_{\frac{1}{4}}^{\frac{3}{4}} s(1-s) p(s) d s \\
& \geq \frac{R}{l} \int_{\frac{1}{4}}^{\frac{3}{4}} s(1-s) p(s) d s=R=\|z\| .
\end{aligned}
$$

Thus, we have $\|T z\| \geq\|z\|, z \in P \cap \partial \Omega_{R}$.

Now, let us choose $\varepsilon>0$ such that

$$
\varepsilon \int_{0}^{1} s(1-s) p(s) d s<1
$$

Then for the above $\varepsilon$, by (H5), there exists $M>R>0$ such that, for any $t \in[0,1]$ and for any $x \geq M$,

$$
f(t, x) \leq \varepsilon x .
$$

Let

$$
R^{*}=\frac{\sigma \int_{0}^{1} s(1-s)\left[p(s)+q_{+}(s)\right] d s+\int_{0}^{1} s(1-s) q_{+}(s) d s}{1-\varepsilon \int_{0}^{1} s(1-s) p(s) d s}+M
$$

where

$$
\sigma=\max _{[0,1] \times[0, M]} f(t, x)+1,
$$

then $R^{*}>M>R$. Let $\Omega_{R^{*}}=\left\{z \in P:\|z\|<R^{*}\right\}$ and $\partial \Omega_{R^{*}}=\left\{z \in P:\|z\|=R^{*}\right\}$. Then, for any $z \in P \cap \partial \Omega_{R^{*}}$, we have

$$
\begin{aligned}
\|T z\| \leq & \int_{0}^{1} s(1-s)\left[p(s) f\left(s,[z(s)-w(s)]^{*}+q_{+}(s)\right) d s\right. \\
\leq & \left\{\left(\max _{[0,1] \times[0, M]} f(t, x)+1\right) \int_{0}^{1} s(1-s)\left[p(s)+q_{+}(s)\right] d s\right. \\
& \left.+\int_{0}^{1} s(1-s)\left[\varepsilon p(s)[z(s)-w(s)]^{*}+q_{+}(s)\right] d s\right\} \\
\leq & \left\{\left(\max _{[0,1] \times[0, M]} f(t, x)+1\right) \int_{0}^{1} s(1-s)\left[p(s)+q_{+}(s)\right] d s\right.
\end{aligned}
$$




$$
\left.+\int_{0}^{1} s(1-s) q_{+}(s) d s\right\}+\varepsilon R^{*} \int_{0}^{1} s(1-s) p(s) d s<R^{*}=\|z\|,
$$

which implies that

$$
\|T z\| \leq\|z\|, \quad z \in P \cap \partial \Omega_{R^{*}} .
$$

By Lemma 2.1, $T$ has two fixed points $z_{1}(t)$ and $z_{2}(t)$ such that

$$
2 r \leq\left\|z_{1}\right\| \leq R \leq\left\|z_{2}\right\| \leq R^{*} .
$$

It follows from $\left\|z_{1}\right\| \geq 2 r$ that

$$
\begin{aligned}
z_{1}(t)-w(t) & \geq\left\|z_{1}\right\| t(1-t)-\int_{0}^{1} G(t, s) q_{-}(s) d s \\
& =\left\|z_{1}\right\| t(1-t)-t(1-t) \int_{0}^{1} q_{-}(s) d s \\
& =\left(\left\|z_{1}\right\|-r\right) t(1-t) \\
& \geq r t(1-t)>0, \quad t \in(0,1),
\end{aligned}
$$

and

$$
\begin{aligned}
z_{2}(t)-w(t) & \geq z_{2}(t)-t(1-t) \int_{0}^{1} q_{-}(s) d s \\
& =z_{2}(t)-t(1-t) r \geq z_{2}(t)-\frac{z_{2}(t)}{\|z\|} r \\
& \geq z_{2}(t)-\frac{r}{R} z_{2}(t) \geq \frac{1}{2} z_{2}(t) \geq \frac{1}{2} t(1-t)\left\|z_{2}\right\| \\
& \geq \frac{R}{2} t(1-t)>0, \quad t \in(0,1) .
\end{aligned}
$$

Let $x_{1}(t)=z_{1}(t)-w(t), x_{2}(t)=z_{2}(t)-w(t)$, then by Lemma 2.2, we have that

$$
x_{1}(t)=z_{1}(t)-w(t)>0, \quad x_{2}(t)=z_{2}(t)-w(t)>0, \quad t \in(0,1)
$$

are two positive solutions of the BVP (1.2). The proof is completed.

Theorem 3.2 Suppose (H1), (H2), (H4) and (H6) hold, and in addition, the following condition is satisfied:

$\left(\mathbf{H}^{*} \mathbf{3}\right)$ There exists a constant $\tilde{R}>\left(\frac{16 R}{3 l}+1\right) L$ such that, for any $(t, x) \in[0,1] \times$ $[0, \tilde{R}]$,

$$
f(t, x) \leq \frac{\tilde{R}}{L}
$$

where $L$ is defined by (H2).

Then the BVP (1.2) has at least two positive solutions $x_{1}(t)$ and $x_{2}(t)$ such that $x_{1}(t) \geq r t(1-t), x_{2}(t) \geq \frac{1}{2} \tilde{R} t(1-t), t \in[0,1]$, where $r$ and $\tilde{R}$ are defined by $(\mathbf{H 2})$ and $\left(\mathbf{H}^{*} \mathbf{3}\right)$, respectively. 
Proof Let $\Omega_{R}=\{z \in P:\|z\|<R\}$ and $\partial \Omega_{R}=\{z \in P:\|z\|=R\}$. Then for any $z \in \partial \Omega_{R}, t \in\left[\frac{1}{4}, \frac{3}{4}\right]$, noticing $R>2 r$, we have

$$
\begin{aligned}
z(t)-w(t) & \geq z(t)-t(1-t) \int_{0}^{1} q_{-}(s) d s=z(t)-t(1-t) r \\
& \geq z(t)-z(t)\|z\| r=z(t)-\frac{r}{R} z(t) \geq \frac{1}{2} z(t) \\
& \geq \frac{1}{2} t(1-t)\|z\| \geq \frac{3}{32} R>0 .
\end{aligned}
$$

So for any $z \in \partial \Omega_{R}, t \in\left[\frac{1}{4}, \frac{3}{4}\right]$, we get

$$
\frac{3}{32} R \leq z(t)-w(t) \leq R
$$

It follows from (H4) and (3.3) that, for any $z \in \partial \Omega_{R}, t \in\left[\frac{1}{4}, \frac{3}{4}\right]$,

$$
\begin{aligned}
\|T z\| \geq(T z)(t) & =\int_{0}^{1} G(t, s)\left[p(s) f\left(s,[z(s)-x(s)]^{*}+q_{+}(s)\right] d s\right. \\
& \geq \int_{\frac{1}{4}}^{\frac{3}{4}} G(t, s)\left[p(s) f(s, z(s)-x(s))+q_{+}(s)\right] d s \\
& \geq \frac{16 R}{3 l} \int_{\frac{1}{4}}^{\frac{3}{4}} G(t, s) p(s) d s \geq \frac{16 R}{3 l} t(1-t) \int_{\frac{1}{4}}^{\frac{3}{4}} s(1-s) p(s) d s \\
& \geq \frac{R}{l} \int_{\frac{1}{4}}^{\frac{3}{4}} s(1-s) p(s) d s=R=\|z\| .
\end{aligned}
$$

Thus, we have $\|T z\| \geq\|z\|, z \in P \cap \partial \Omega_{R}$.

Next, let $\Omega_{\tilde{R}}=\{z \in P:\|z\|<\tilde{R}\}$ and $\partial \Omega_{\tilde{R}}=\{z \in P:\|z\|=\tilde{R}\}$. Then for any $z \in \partial \Omega_{\tilde{R}}, s \in[0,1]$, we have

$$
\tilde{R}>R>2 r>0, \quad \frac{\tilde{R}}{L}-1>\frac{16 R}{3 l},
$$

and

$$
0 \leq[z(s)-w(s)]^{*} \leq z(s) \leq\|z\| \leq \tilde{R}
$$


It follows from $\left(\mathbf{H}^{*} \mathbf{3}\right)$ that

$$
\begin{aligned}
\|T z\| & \left.=\max _{t \in[0,1]} \int_{0}^{1} G(t, s)\left[p(s) f\left(s,[z(s)-w(s)]^{*}\right)\right)+q_{+}(s)\right] d s \\
& \leq \int_{0}^{1} s(1-s)\left[\frac{\tilde{R}}{L}-1\right]\left[p(s)+q_{+}(s)\right] d s \\
& \leq \frac{\tilde{R}}{L} \int_{0}^{1} s(1-s)\left[p(s)+q_{+}(s)\right] d s \\
& =\tilde{R}=\|z\| .
\end{aligned}
$$

Therefore, $\|T z\| \leq\|z\|, z \in P \cap \partial \Omega_{\tilde{R}}$.

On the other hand, choose constants $\alpha, \beta$ and a real number $K>0$ such that

$$
[\alpha, \beta] \subset(0,1), \quad 1 \leq \frac{1}{2} \beta^{2}(1-\alpha)^{2} K \int_{\alpha}^{\beta} s(1-s) p(s) d s
$$

From (H6), there exists $R_{1}>\tilde{R}$ such that, for any $t \in[\alpha, \beta]$,

$$
f(t, x) \geq K x, \quad x \geq R_{1}
$$

Take $R^{*}=\max \left\{\frac{2 R_{1}}{\beta(1-\alpha)}, R_{1}\right\}$, then $R^{*}>R_{1}>\tilde{R}>R>2 r$. Let $R^{*}=\{z \in P:\|z\|<$ $\left.R^{*}\right\}$, then for any $z \in P \cap \partial \Omega_{R^{*}}$ and for any $t \in[\alpha, \beta]$, we have

$$
\begin{aligned}
z(t)-w(t) & \geq z(t)-t(1-t) \int_{0}^{1} q_{-}(s) d s \\
& =z(t)-t(1-t) r \\
& \geq z(t)-z(t)\|z\| r=z(t)-\frac{r}{R^{*}} z(t) \\
& \geq \frac{1}{2} z(t) \geq \frac{1}{2} t(1-t)\|z\| \geq \frac{1}{2} \beta(1-\alpha) R^{*} \\
& \geq R_{1}>0
\end{aligned}
$$

Therefore from (3.4)-(3.5), for any $z \in P \cap \partial \Omega_{R^{*}}$ and $t \in[\alpha, \beta]$, we have

$$
\begin{aligned}
(T z)(t) & =\int_{0}^{1} G(t, s)\left[p(s) f\left(s,[z(s)-w(s)]^{*}\right)+q_{+}(s)\right] d s \\
& \geq \int_{0}^{1} G(t, s) p(s) f\left(s,[z(s)-w(s)]^{*}\right) d s \\
& \geq \int_{\alpha}^{\beta} G(t, s) p(s) f\left(s,[z(s)-w(s)]^{*}\right) d s
\end{aligned}
$$




$$
\begin{aligned}
& \left.\geq \int_{\alpha}^{\beta} G(t, s) p(s) K[z(s)-w(s)]^{*}\right) d s \\
& =\int_{\alpha}^{\beta} G(t, s) p(s) K[z(s)-w(s)] d s \\
& \geq \frac{1}{2} \beta(1-\alpha) K R^{*} \int_{\alpha}^{\beta} G(t, s) p(s) d s \\
& \geq \frac{1}{2} \beta(1-\alpha) K R^{*} t(1-t) \int_{\alpha}^{\beta} s(1-s) p(s) d s \\
& \geq \frac{1}{2} \beta^{2}(1-\alpha)^{2} K R^{*} \int_{\alpha}^{\beta} s(1-s) p(s) d s \geq R^{*} .
\end{aligned}
$$

Thus, $\|T z\| \geq\|z\|, z \in P \cap \partial \Omega_{R^{*}}$.

By Lemma 2.1, $T$ has two fixed points $z_{1}(t)$ and $z_{2}(t)$ such that

$$
2 r \leq\left\|z_{1}\right\| \leq \tilde{R} \leq\left\|z_{2}\right\| \leq R^{*} .
$$

It follows from $\left|z_{1}\right| \geq 2 r$ that

$$
\begin{aligned}
z_{1}(t)-w(t) & \geq\left\|z_{1}\right\| t(1-t)-\int_{0}^{1} G(t, s) q_{-}(s) d s \\
& =\left\|z_{1}\right\| t(1-t)-t(1-t) \int_{0}^{1} q_{-}(s) d s \\
& =\left(\left\|z_{1}\right\|-r\right) t(1-t) \\
& \geq r t(1-t)>0, \quad t \in(0,1)
\end{aligned}
$$

and

$$
\begin{aligned}
z_{2}(t)-w(t) & \geq z_{2}(t)-t(1-t) \int_{0}^{1} q_{-}(s) d s \\
& =z_{2}(t)-t(1-t) r \geq z_{2}(t)-z_{2}(t)\|z\| r \\
& =z_{2}(t)-\frac{r}{\tilde{R}} z_{2}(t) \geq \frac{1}{2} z_{2}(t) \geq \frac{1}{2} t(1-t)\left\|z_{2}\right\| \\
& \geq \frac{1}{2} t(1-t) \tilde{R}>0, \quad t \in(0,1) .
\end{aligned}
$$

Let $x_{1}(t)=z_{1}(t)-w(t), x_{2}(t)=z_{2}(t)-w(t)$, then by Lemma 2.2, we have that

$$
x_{1}(t)=z_{1}(t)-w(t)>0, \quad x_{2}(t)=z_{2}(t)-w(t)>0, \quad t \in(0,1)
$$

are two positive solutions of the BVP (1.2). The proof is completed. 


\section{Applications}

Example 4.1 Consider the following singular semipositone boundary value problem

$$
\left\{\begin{array}{l}
-x^{\prime \prime}=\frac{f(t, x)}{t(1-t)}-\frac{1}{\sqrt{t}}, \quad t \in(0,1), \\
x(0)=0, \quad x(1)=0,
\end{array}\right.
$$

where

$$
f(t, x)= \begin{cases}\frac{1}{2} x, & 0 \leq x \leq 4 \\ 349 x-1394, & 4 \leq x \leq 6, \\ x+694, & 6 \leq x \leq 64 \\ -\frac{187}{9} x+\frac{18790}{9}, & 64 \leq x \leq 100, \\ x^{\frac{1}{2}}, & x \geq 100 .\end{cases}
$$

The BVP (4.1) has at least two positive solutions $x_{1}(t)$ and $x_{2}(t)$ such that

$$
4 \leq\left\|x_{1}\right\| \leq 64 \leq\left\|x_{2}\right\|,
$$

and

$$
x_{1}(t) \geq 2 t(1-t), \quad t \in[0,1], \quad x_{2}(t) \geq 8 t(1-t), \quad t \in[0,1] .
$$

Proof In fact, let

$$
p(t)=\frac{1}{t(1-t)}, \quad q_{+}(0) \equiv 0, \quad q_{-}(t)=\frac{1}{\sqrt{t}},
$$

then

$$
r=\int_{0}^{1} \frac{1}{\sqrt{s}} d s=2, \quad L=\int_{0}^{1} s(1-s)\left[p(s)+q_{+}(s) d s\right]=1<2 r=4 .
$$

For any $(t, x) \in[0,1] \times[0,4]$, we have

$$
f(t, x) \leq 2 \leq \frac{2 r}{L}-1=3 .
$$

So (H1)-(H3) hold.

Next, let $R=64$, then $R>2 r=4, l=\int_{\frac{1}{4}}^{\frac{3}{4}} s(1-s) p(s) d s=\frac{1}{2}$. Thus, for any $(t, x) \in[0,1] \times[6,64]$, we have

$$
f(t, x) \geq 700 \geq \frac{16 R}{3 l}=\frac{32 \times 64}{3} \approx 682.7,
$$

and

$$
\lim _{x \rightarrow+\infty} \frac{f(t, x)}{x}=\lim _{x \rightarrow+\infty} x^{-\frac{1}{2}}=0 .
$$


Therefore (H4)-(H5) hold. By Theorem 3.1, the BVP (4.1) has at least two positive solutions $x_{1}(t)$ and $x_{2}(t)$ such that

$$
4 \leq\left\|x_{1}\right\| \leq 64 \leq\left\|x_{2}\right\|,
$$

and

$$
x_{1}(t) \geq 2 t(1-t), \quad t \in[0,1], \quad x_{2}(t) \geq 8 t(1-t), \quad t \in[0,1] .
$$

Example 4.2 Consider the following singular semipositone boundary value problem

$$
\left\{\begin{array}{l}
-x^{\prime \prime}=\frac{f(x)}{3 t(1-t)}-\frac{1}{2+3 \sqrt[3]{4}}\left\{\frac{1}{\sqrt{t}}+\frac{1}{\sqrt[3]{\left(t-\frac{1}{2}\right)^{2}}}\right\}, \quad t \in(0,1), \\
x(0)=0, \quad x(1)=0 .
\end{array}\right.
$$

where

$$
f(x)= \begin{cases}x^{\frac{1}{2}}+x^{2}, & 0 \leq x \leq 1 \\ 511 x-509, & 1 \leq x \leq 3 \\ \frac{76}{29} x+\frac{29468}{29}, & 3 \leq x \leq 32 \\ \frac{25}{184} x+\frac{25200}{23}, & 32 \leq x \leq 400 \\ \frac{x^{2}}{400}+750, & x \geq 342\end{cases}
$$

The BVP (4.3) has at least two positive solutions $x_{1}(t)$ and $x_{2}(t)$ such that

$$
x_{1}(t) \geq t(1-t), \quad t \in[0,1], \quad x_{2}(t) \geq 200 t(1-t), \quad t \in[0,1] .
$$

Proof In fact, Let

$$
p(t)=\frac{1}{3 t(1-t)}, \quad q_{+}(0) \equiv 0, \quad q_{-}(t)=\frac{1}{2+3 \sqrt[3]{4}}\left\{\frac{1}{\sqrt{t}}+\frac{1}{\sqrt[3]{\left(t-\frac{1}{2}\right)^{2}}}\right\},
$$

then

$$
\begin{aligned}
& r=\int_{0}^{1} q_{-}(s) d s=1, \quad l=\int_{\frac{1}{4}}^{\frac{3}{4}} s(1-s) \frac{1}{3 s(1-s)} d s=\frac{1}{6}, \\
& L=\int_{0}^{1} s(1-s)\left[p(s)+q_{+}(s) d s\right]=\frac{1}{3}<2 r=2 .
\end{aligned}
$$

Let $R=32>2 r=2$, then for any $x \in[3,32]$, we have

$$
f(x) \geq \frac{16 R}{3 l}=1024 .
$$


On the other hand, let $\tilde{R}=400>\left(\frac{16 R}{3 l}+1\right) L=\frac{1025}{3}$, then, for any $x \in[0,400]$,

$$
f(t, x) \leq 1150 \leq 1199=\frac{\tilde{R}}{L}-1 .
$$

Obviously,

$$
\lim _{x \rightarrow+\infty} \frac{f(t, x)}{x}=+\infty
$$

for $t$ uniformly holds on any close subinterval of $(0,1)$. It follows from Theorem 3.2 that the BVP (4.3) has at least two positive solutions $x_{1}(t)$ and $x_{2}(t)$ such that

$$
x_{1}(t) \geq t(1-t), \quad t \in[0,1], \quad x_{2}(t) \geq 200 t(1-t), \quad t \in[0,1] .
$$

Remark 4.1 In Example 4.2, we notice that the nonlinearity is singular at $t=0$ and $t=\frac{1}{2}$, which shows that the singularity of the semipositone boundary value problem (1.2) may occur not only at the endpoints of the interval $(0,1)$, but also in the internal area of the interval $(0,1)$. Moreover, since $q:(0,1) \rightarrow[0,+\infty)$ only needs to be Lebesgue integrable, this implies that $q$ may be singular at some zero measure set of $(0,1)$.

Open Access This article is distributed under the terms of the Creative Commons Attribution Noncommercial License which permits any noncommercial use, distribution, and reproduction in any medium, provided the original author(s) and source are credited.

\section{References}

1. Agarwal, R., O'Regan, D.: Nonlinear superlinear singular and nonsingular second order boundary value problems. J. Differ. Equ. 143, 60-95 (1998)

2. Anuradha, V., Hai, D., Shivaji, R.: Existence results for superlinear semipositone BVP's. Proc. Am. Math. Soc. 124, 757-746 (1996)

3. Aris, R.: Introduction to the Analysis of Chemical Reactors. Englewood Cliffs, Prentice-Hall (1965)

4. Bai, Z.: The method of lower and upper solutions for a bending of an elastic beam equation. J. Math. Anal. Appl. 248, 195-202 (2000)

5. Castro, A., Maya, C., Shivaji, R.: Nonlinear eigenvalue problems with semipositone. Electron. J. Differ. Equ. Conf. 05, 33-49 (2000)

6. Guo, D., Lakshmikantham, V.: Nonlinear Problems in Abstract Cone. Academic Press, New York (1988)

7. Ha, K., Lee, Y.: Existence of multiple positive solutions of singular boundary value problems,. Nonlinear Anal. 28, 1429-1438 (1997)

8. Lan, K., Webb, J.: Positive solutions of semilinear differential equations with singularities. J. Differ. Equ. 148, 407-421 (1998)

9. Liu, Y.: Global structure of solutions for a class of two-point boundary value problems involving singular and convex or concave nonlinearities. J. Math. Anal. Appl. 322, 75-86 (2006)

10. Ma, R., Zhang, J., Fu, S.: The method of lower and upper solutions for fourth-order two point boundary value problems. J. Math. Anal. Appl. 215, 415-422 (1997)

11. Wei, Z.: Positive solution of singular Dirichlet boundary value problems for second order differential equation system. J. Math. Anal. Appl. 328, 1255-1267 (2007)

12. Zhang, X., Liu, L.: Positive solutions of superlinear semipositone singular Dirichlet boundary value problems. J. Math. Anal. Appl. 316, 525-537 (2006) 